\title{
SÍNDROME DAS APNÉIAS / HIPOPNÉIAS OBSTRUTIVAS DO SONO (SAHOS)
}

\author{
OBSTRUCTIVE SLEEP APNEA/HYPOPNEA SYNDROME
}

Geruza A Silva1', Leonardo A T Giacon²

\begin{abstract}
${ }^{1}$ Docente. Disciplina de Pneumologia. Departamento de Clínica Médica.Faculdade de Medicina de Ribeirão Preto - USP. ${ }^{2}$ Médico Pneumologista. Faculdade de Medicina de Ribeirão Preto - USP

Correspondência: Profa Dra. Geruza Alves da Silva.

Departamento de Clínica Médica. Hospital das Clínicas da Faculdade de Medicina de Ribeirão Preto-USP.

Av. dos Bandeirantes, 3900. Bairro Monte Alegre. CEP: 14048-900 - Ribeirão Preto-SP

E-mail: gadsilva@fmrp.usp.br
\end{abstract}

Silva GA, Giacon LAT. Síndrome das Apnéias/Hipopnéias Obstrutivas do Sono (SAHOS). Medicina (Ribeirão Preto) 2006; 39 (2): 185-194.

RESUMO: Os distúrbios respiratórios do sono constituem um tópico relativamente novo do conhecimento científico, cujo manejo envolve a participação de diversas Disciplinas da área Médica. Sua importância tem crescido desde a disponibilização em escala "democrática" dos recursos diagnósticos e terapêuticos que se iniciou no começo dos anos de 1980. Isso permitiu constatações sucessivas do papel do sono e dos seus distúrbios no aumento da morbidade e da mortalidade. A prevalência também tem aumentado desde os primeiros levantamentos epidemiológicos em adultos, em parte devido ao aumento dos fatores de risco, principalmente a obesidade e idade, mas também ao melhor reconhecimento de anormalidades devido ao aumento na sensibilidade dos métodos diagnósticos utilizados. As apnéias/hipopnéias do sono têm se revelado ser o mais importante e freqüente distúrbio respiratório do sono, constituindo cerca de $80 \%$ dos casos que procuram as Clínicas dedicadas a estudar as anormalidades do sono.

Descritores: Apnéia. Sono. Ronco. Distúrbios do Sono por Sonolência Excessiva. Síndromes da Apnéia do Sono. Apnéia do Sono Tipo Obstrutiva.

\section{1- INTRODUÇÃO}

Sinonímia: Síndrome das Apnéias do Sono (SAS) (SAOS)

Síndrome das Apnéias Obstrutivas do Sono

A síndrome das apnéias/hipopnéias do sono tem se revelado ser o mais importante e freqüente distúrbio respiratório do sono. Decorre de oclusão ou semioclusão repetidas das Vias Aéreas Superiores (VAS) durante o sono produzindo pausas na respiração, de duração variável. O distúrbio é comum e acomete to- das as faixas etárias e ambos os sexos, independentemente do peso, embora seja mais freqüente nos obesos ou sobrepeso.

O interesse pelas apnéias do sono ganhou vulto a partir do final dos anos de 1970 após 30 anos de significantes estudos e descobertas que alavancaram a importância do sono e seus distúrbios, sobre a saúde.

\section{1- Notas históricas}

Data do final dos anos de 1920 a descoberta da possibilidade de registro de ondas de atividade cere- 
bral na vigília e no sono, processo que foi denominado de eletroencefalograma, o qual desencadeou um real interesse científico pelo sono. O recurso permitiu na década seguinte, caracterizar-se o sono humano pela ocorrência de ondas de atividade neuronal, grandes e lentas e de fusos, que o diferenciavam da vigília pelo registro de ondas pequenas e ritmo alfa ${ }^{1}$. Porém, por mais alguns anos manteve-se o conceito errôneo de que o sono é um estado passivo, de inatividade decorrente da diminuição de estímulos ambientais. Muito dos equívocos que persistiram a respeito da caracterização do sono após 1928, deve-se possivelmente, ao fato de que os registros do sono eram feitos por amostragem. O registro contínuo era considerado desnecessário, extravagante e caro. A necessidade do registro contínuo do sono, durante toda a noite, para exata avaliação desse estado e das ocorrências fisiológicas e patológicas a ele relacionadas, ficou evidente somente na década de 1950 com os estudos de Aserinsky e Kleitman ${ }^{2}$ que resultaram na descoberta do sono com movimentos rápidos dos olhos ou sono REM. Estudos subsequientes de Dement e Kleitman ${ }^{3}$, utilizando o registro contínuo do sono, evidenciaram variação periódica do padrão eletroencefalográfico, que permitiram caracterizar o sono como um fenômeno ativo e não-homogêneo, subdividido em estágios superficiais e profundos, que se repetem ciclicamente, com duração de 90-100 minutos, a contar do final de um período de REM até o final do próximo (ver Capítulo I sobre sono normal).

$\mathrm{Na}$ seqüência, importantes descobertas foram feitas no campo dos distúrbios do sono, ressaltandose: a justificativa para a atonia muscular da Cataplexia, a relação entre baixa latência ao REM e depressão endógena e o reconhecimento da hipersonolência como sendo o mais frequiente e danoso distúrbio do sono.

No que se refere às pausas respiratórias durante o sono, existem relatos já na literatura grega antiga e na literatura britânica de Shakespeare, de que elas eram observadas à beira do leito, mas foi somente em 1965, que a descrição de apnéias obstrutivas durante o sono revestiu-se de cunho científico. Neste ano dois grupos independentes, Gastaut et al. ${ }^{4}$ na França e Jung e Ruho ${ }^{5}$, na Alemanha publicaram seus achados documentados que constituem os primeiros registros de apnéia obstrutiva do sono, em humanos. Na Itália, um grupo liderado por Lugaresi et al. ${ }^{6}$ proporciou uma descrição completa da síndrome das apnéias do sono, incluindo a primeira observação de apnéia obstrutiva em paciente magro, a descrição da correlação com manifestações cardiovasculares, e uma clara identifi- cação da importância do ronco e da hipersonolência como indicadores diagnósticos.

Em 1972 a Clínica de Sono de Stanford adicionou ao seu campo de pesquisas sobre o sono, dois elementos de fundamental importância para a consolidação dos estudos sobre os distúrbios do sono que originaram os primeiros conceitos organizados, sobre a síndrome das apnéias do sono, que inclui sua relação estreita com a hipersonolência diurna ${ }^{7}$. A primeira adição foi a do médico francês Christian Guilleminault (um neurologista e psiquiatra com conhecimento e experiência com os estudos Europeus sobre apnéias do sono) e a segunda adição foi a monitorização cardíaca e respiratória ao registro do sono, processo que foi denominado de polissonografia em 1974, pelo Dr. Jerome Holland, também pertencente ao grupo.

No início dos anos de 1980, em cima da consciência consolidada acerca da importância das apnéias do sono, foram propostas duas formas de tratamento, destinadas a abolir as apnéias obstrutivas do sono, deslocando para segundo plano o emprego da traqueostomia, processo eficiente, porém indesejado, de manejo terapêutico da síndrome: a uvulopalatofaringoplastia proposta por Fujita et al. ${ }^{8}$, logo ficou restrita a situações bem específicas (ver Capítulo VII sobre tratamento cirúrgico da oclusão das vias aéreas superiores); já o uso de pressão positiva contínua nas vias aéreas (CPAP), proposto pelo Pneumologista Australiano, Colin Sullivan et al. ${ }^{9}$, revelou-se muito eficaz para tratamento geral dos distúrbios respiratórios do sono e amplamente aceito por pacientes e médicos.

Atualmente novas técnicas não-invasivas de tratamento estão disponíveis e servem como alternativa para aliviar sintomas em alguns pacientes que não se adaptam ou não aceitam o CPAP. Técnicas cirúrgicas que se destinam a desimpedir as VAS, seja removendo obstáculos anatômicos, seja reestruturando os ossos da face, também foram desenvolvidas ou adaptadas para tratamento da oclusão das vias aéreas durante o sono.

\section{2- PREVALÊNCIA E FATORES DE RISCO}

A SAHOS é atualmente considerada um problema de saúde pública. A prevalência varia com a faixa etária e com critérios utilizados para diagnosticar a síndrome, por exemplo: os diversos estudos de prevalência utilizaram diferentes índices de apnéia/ hipopneia, desde 5 até 15 eventos por hora de sono, associado ou não a sintomas diurnos de sonolência. 
O distúrbio corre principalmente nos homens, obesos, de meia idade, mas atinge todas as faixas etárias e ambos sexos, bem como os indivíduos de peso normal e os magros. Nas mulheres pós-menopausa, que não fazem reposição hormonal, torna-se tão freqüente quanto nos homens e o uso de hormônios nesta fase está associado a baixa prevalência.

Os riscos para desenvolver distúrbio respiratório do sono aumentam com a idade e com o peso corporal sendo que nos mais velhos (mais que 65 anos) o significado clínico diminui junto com a prevalência.

Outro fator que influencia a prevalência é o tipo de população estudada. Porém de um modo geral, os resultados dos principais estudos epidemiológicos de Israel, Bolonha, Scandinávia e Zaragoza todos convergem no mesmo sentido dos resultados obtidos nos dois maiores estudos realizados nos Estados Unidos ${ }^{10,11}$ .Eles permitem estimar a prevalência geral como sendo de $4 \%$ (3\% a $8 \%)$ nos homenas e $2 \%$ nas mulheres na faixa etária de 40 aos 65 anos de idade, e que a prevalência absoluta mínima de SAOS clinicamente significante é de $1 \%$.

\section{3- CONCEITOS}

De acordo com as propostas da American Academy of Sleep Medicine Task Force ${ }^{12}$ de 1999:

- as apnéias obstrutivas do sono compreendem pausas repetidas na respiração, com duração maior ou igual a 10 segundos, seguidas por um despertar transitório. Devem acompahar-se de queda de mais de 3 pontos na saturação da oxihemoglobina.

A visão atual tende a acrescentar mais rigor ao conceito, eliminando a necessidade de dessaturação da hemoglobina e de despertar, ainda que transitório, pós-apnéia.

Durante o evento os movimentos do tórax e do abdômen podem tornar-se opostos (a parede abdominal se retrai enquanto a caixa torácica se expande, a cada tentativa frustrada de respirar contra a via aérea ocluída) e o fluxo aéreo fica reduzido a menos de $20 \%$, ou completamente ausente.

Quase sempre as apnéias obstrutivas acompanham-se de hipopnéias e, menos freqüentemente, de outros tipos de apnéias, centrais e mistas.

As hipopnéias do sono compreendem períodos repetidos de redução de até $80 \%$ do fluxo aéreo acompanhados ou não, de queda de pelo menos 3 pontos na saturação da hemoglobina. Se o fluxo cai mais que $50 \%$ não precisa haver queda na saturação. Ao final de cada evento ocorre um despertar transitório ou superficialização do sono. As hipopnéias podem ocorrer sozinhas ou acompanhadas de roncos, ou ainda, intercaladas aos episódios de apnéias (mais comum).

A definição de apnéia e hipopnéia proposta pelo "Sleep Heart Health Study" (resultado de um acompanhamento longitudinal de 6000 indivíduos envolvidos em estudos de doença cardiovascular) ${ }^{13}$, requer sempre uma dessaturação de $4 \%$ ou mais e não inclui nenhuma medida de descontinuidade do sono (despertares ou superficialização do sono). Esses critérios foram adotados largamente porque se mostraram preditivos de seqüela cardiovascular, e muito reprodutíveis.

Em resumo, num episódio de apnéia do sono registram-se as seguintes alterações:

- o fluxo aéreo cessa (porque a faringe oclui);

- os movimentos do tórax e do abdômen ficam diminuídos e/ou opostos (paradoxais);

- a saturação da oxihemoglobina cai;

- ocorre bradicardia durante a apnéia e taquicardia, em seguida;

- ocorre um despertar transitório ou superficialização do sono após a apnéia;

- o episódio dura 10 segundos ou mais.

Na SAHOS os episódios de apnéias e hipopnéias ocorrem numa frequência de 5 até 100 (ou mais) por hora de sono. Essa frequiência é chamada de Índice de Distúrbio Respiratório (IDR) ou Índice de Apnéias-Hipopnéias (IAH), e constitui o principal indicador de gravidade do distúrbio.

\section{4- FISIOPATOLOGIA}

A oclusão das VAS durante o sono ocorre ao nível da faringe, principalmente orofaringe, por colapso decorrente do relaxamento das estruturas musculares que revestem o canal, em face da pressão negativa intraluminal inspiratória. Cerca de 24 músculos participam das funções da faringe que incluem a deglutição, a fala e a respiração. Seis desses músculos (o mais bem estudado é o genioglosso) têm como principal papel a manutenção da permeabilidade da faringe. Durante o sono a atividade motora do conjunto muscular fica muito diminuída ou mesmo abolida, reduzindo sensivelmente a luz do canal e a passagem de ar. Qualquer fator adicional, anormal, de redução do calibre da via aérea que se sobreponha a essa situa- 


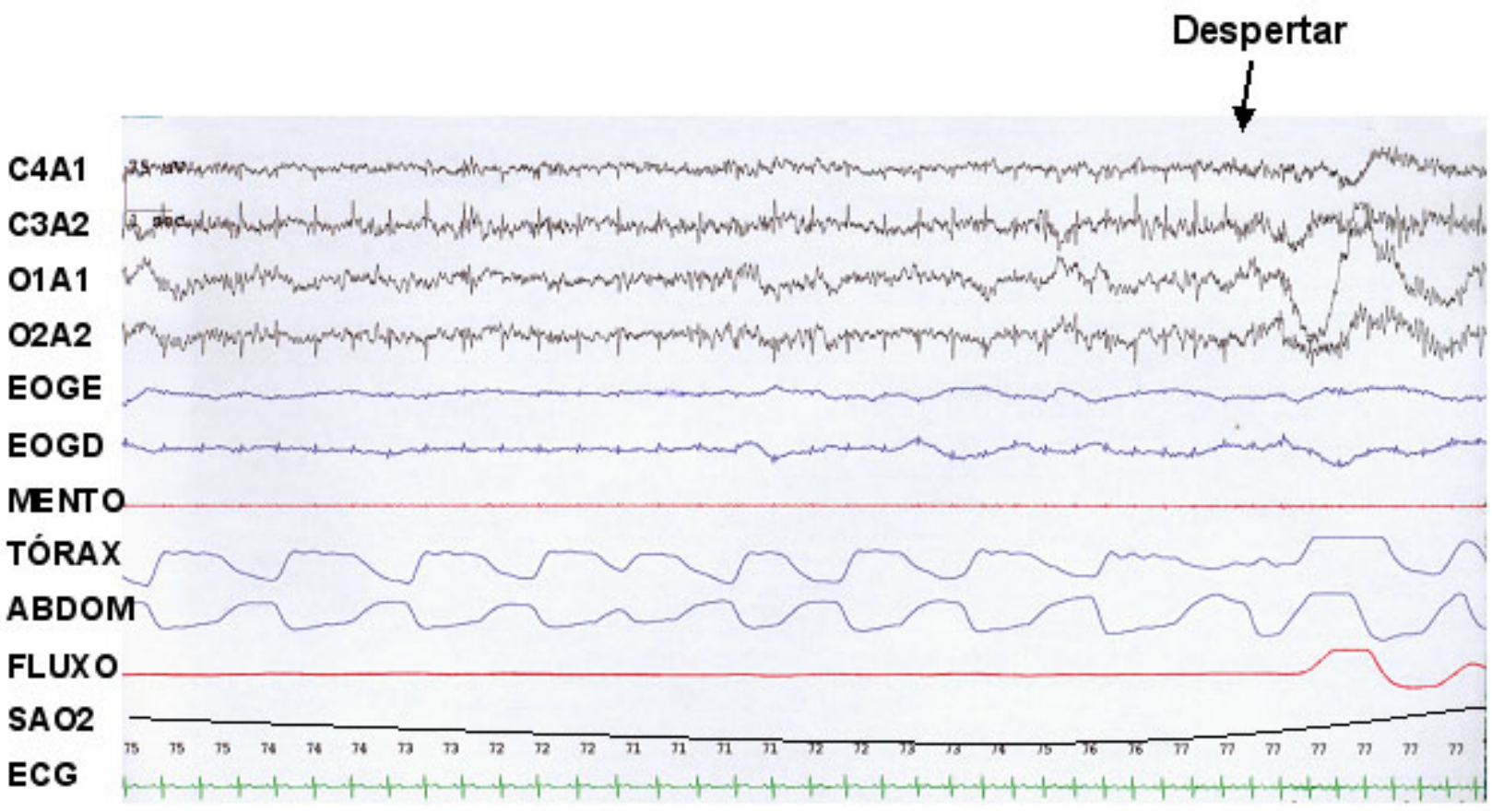

Figura 1: O gráfico é a representação de uma "época" de 30 segundos de exame polissonográfico; nos últimos segundos, após um despertar, os traçados dos movimentos do tórax, do abdomem e do fluxo aéreo desenham o mesmo trajeto sinusoide, em fase, (respiração normal) seguindo-se ao traçado de uma apnéia obstrutiva evidenciada por ausência de deflexão da linha do fluxo e, movimentos torácico e abdominal em oposição de fase. A saturação $\left(\mathrm{SAO}_{2}\right)$ cai ao longo da pausa respiratória e a freqüência cardíaca sofre flutuações.

ção fisiológica, favorece o colapso durante o sono. No sono, mesmo o mais importante estímulo local para ativação dos músculos, que é a pressão negativa intraluminal é incapaz de impedir o colapso. Esse efeito do estado de sono é provavelmente mediado por perda de reflexo neuromuscular.

Mecanismo de oclusão das VAS no sono: o estímulo inspiratório que parte dos centros respiratórios e chega aos músculos principais da respiração, diafragma e intercostais, faz dilatar a caixa torácica, processo que não se acompanha de imediato enchimento pulmonar, por causa da redução da passagem do ar através das vias aéreas demasiadamente estreitas. A pressão negativa que se forma no interior das vias aéreas aspira as paredes da faringe na direção central do tubo faringeano, determinando o colapso. Uma justificativa adicional para o fechamento das VAS é a de que os músculos dilatadores da faringe, na SAHOS podem ser lesados e fatigados pelo colapso repetitivo; pode ocorrer edema e subseqüente dano tecidual decorrente desse processo de sucção, alem de trauma pelo ronco, agravando e perpetuando as apnéias.

A redução da luz faringeana a um nível crítico capaz de colapsar durante o sono, tem múltiplas etiologias, por exemplo: a) defeitos anatômicos na própria faringe como amígdalas ou adenóides volumosas, úvula comprida, macroglossia com aumento da base da língua, redundância de palato, depósitos de gordura parafaringeana ou mixedema, deformidades orofaciais como mandíbula pequena e retrognata ou maxila retraída; b) defeitos anatômicos nasais como desvios de septo, pólipos e obstrução por congestão; c) síndromes que afetam o calibre das vias aéreas como a trissomia 21, acondroplasia, mucopolissacaridoses, síndrome de Marfan e outras. Essas anomalias pouco ou nada interferem com a manutenção da respiração normal no estado de vigília que é assegurada pela eficiente atividade motora dos músculos, aumentada nos pacientes com empecilhos nas vias aéreas ${ }^{14 / 17}$. O prejuízo muscular é só no sono, o que condiz com o conceito de que SAHOS é um distúrbio respiratório determinado pelo sono. 

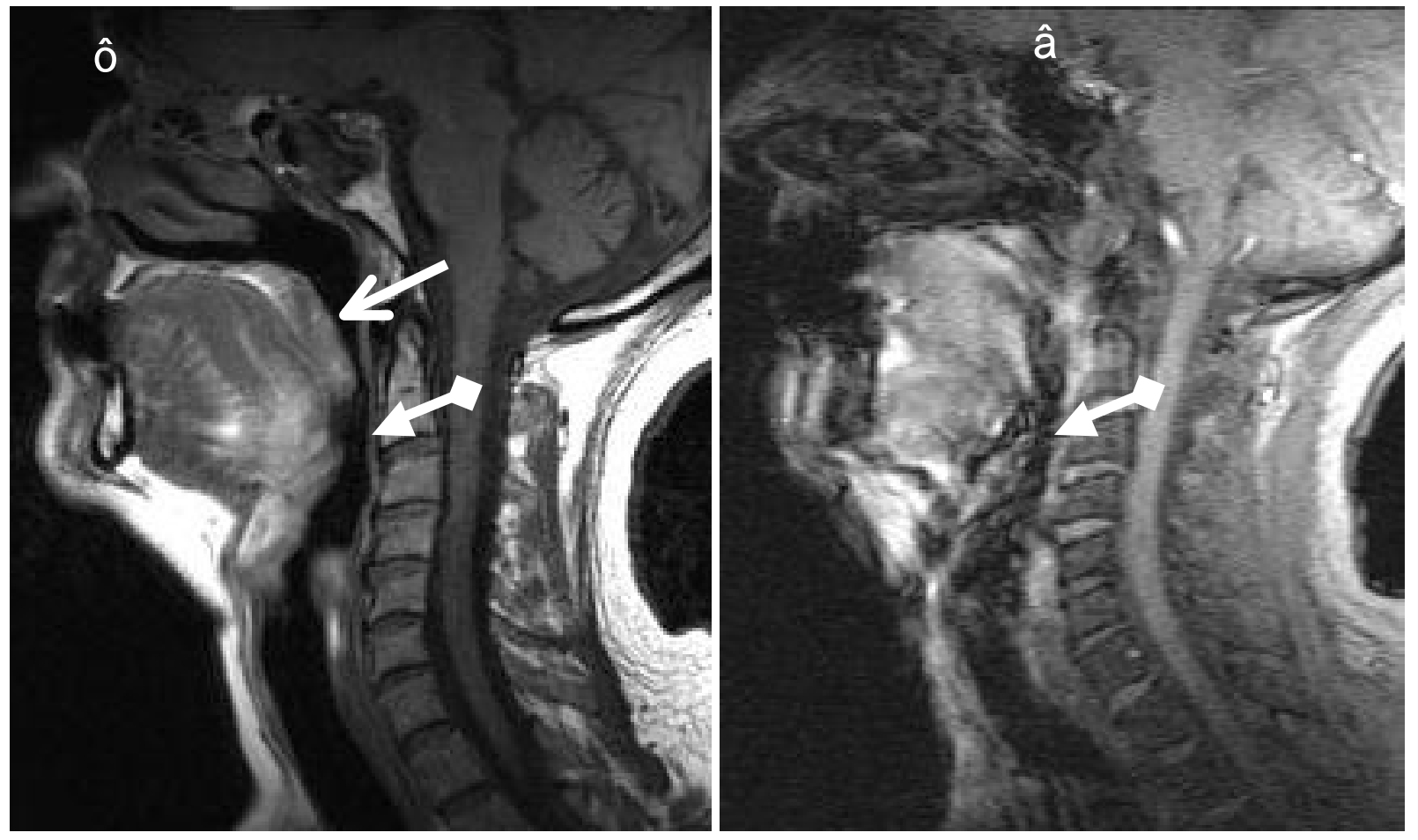

Figura 2: Ressonância magnética de região faringeana em paciente com retrognatia e retroposição da língua; palato mole redundante medindo $43 \mathrm{~mm}$ (seta superior, A), comprometendo o espaço aéreo da nasofaringe e orofaringe (setas, A). Conclusão: redução do espaço aéreo da orofaringe no repouso por aumento de partes moles e palato redundante (A), com obliteração durante o sono induzido (seta, B). Fonte: Serviço de Imagem do HCFMRP-USP, nov/2005.

\section{5- COMO TERMINA UM EPISÓDIO DE APNÉIA DO SONO}

Uma pausa na respiração (apnéia) produz hipoxia, hipercapnia e elevação da concentração de íons $\mathrm{H}^{+}$que vão estimular os quimiorreceptores centrais do tronco cerebral e os periféricos, da crossa da aorta e seios carotídeos; os estímulos crescentes sobre os músculos respiratórios, fazem aumentar o esforço muscular respiratório provocando um despertar. O despertar devolve o tônus dos músculos dilatadores da faringe desobstruindo-a. Isso faz cair a resistência das vias aéreas e o individuo retoma a ventilação ${ }^{18}$. É importante salientar que mesmo na ausência desse estímulo adicional durante uma apnéia, o esforço respiratório contra a via aérea ocluída, constitui o principal elemento promotor do despertar. Enfatizamos que, embora a atividade dos músculos dilatadores da faringe aumente na medida em que uma apnéia progride, o aumento é geralmente insuficiente para restabelecer a permeabilidade da faringe ${ }^{14,15}$. Então o paciente acor- da ou superficializa o sono, repetidamente para sair das apnéias.

\section{6- QUADRO CLÍNICO}

Os diversos efeitos danosos, diretamente produzidos pelas apnéias/hipopnéias sobre o organismo, que são ${ }^{7,14,16,18}$ :

- Hipoxemia

- Reoxigenação

- Hipercapnia

- Acidose

- Oscilações da pressão intratorácica

- Alterações do sistema nervoso autônomo

- Fragmentação do sono por despertares excessivos

- Deprivação de sono profundo (estágios 3 e 4 do sono NREM) e de REM, levam a manifestações clínicas significantes que se estabelecem à longo prazo.

A manifestação clínica mais objetiva e constante no paciente que geralmente procura diagnóstico 
a partir de sugestões de amigos, de familiares ou da mídia, é a sonolência diurna. Com frequiência ele não consegue caracterizar seu estado de quase "torpor" como sendo sonolência, mesmo quando mantém os olhos semicerrados durante a consulta. A maior parte deles, entretanto, apresenta "score" alto nas escalas de avaliação de sonolência (10 pontos ou mais na escala de Epworth ${ }^{19}$, por exemplo). A sonolência acarreta baixo desempenho intelectual e físico e embaraços sociais pela desatenção, falta de memória e indiferença geradas, expõe o paciente e circunstantes a riscos de acidentes, e produz sensação progressiva de cansaço. Já foi demonstrado que alguns pacientes, após tratamento e sem sonolência, mantêm anormalidades cognitivas associadas a alterações permanentes na memória. Alterações do humor tais como, irritabilidade e depressão também são freqüentemente encontradas.

Outro sintoma comum é o ronco; este é produzido pela vibração das estruturas moles da faringe decorrente do baixo fluxo de ar pela via aérea estreita. Pode ser alto o suficiente para expulsar (por incômodo) o parceiro de quarto e até de outros compartimentos da casa. O ronco é tipicamente interromopido pelas pausas respiratórias. Alguns pacientes muito comprometidos por apnéias do sono não têm tempo para roncar, pois ficam sem fluxo aéreo durante todo o tempo de sono.

Sintomas mais gerais comumente presentes são: polaciúria por causa da produção exagerada do hormônio natriurético atrial estimulada pelo estiramento do átrio submetido às variações de pressão intratorácicas; cefaléia latejante matinal e nos despertares noturnos, obstrução nasal ao despertar, boca seca e dor de garganta ao despertar, ganho recente de peso, história de hipertensão arterial sistêmica ${ }^{13,20}$, de acidente vascular cerebral e de doença coronariana ${ }^{13,21}$.

O sono pode ser agitado por movimentação excessiva no leito e pernas inquietas, sonilóquio, tosse e engasgos e despertares bruscos por sensação de sufocação; as pausas na respiração (apnéias) podem ser observadas por familiares que, por vezes, até cronometram a duração dos episódios. As apnéias do sono podem desencadear parassonias, tais como o sonambulismo, por exemplo, e o tratamento das apnéias pode diminuir ou acabar com a parassonia (ver Capítulo V sobre parassonias e outros distúrbios do sono, associados).

A ocorrência da sensação de sufocação noturna exige que se afaste o diagnóstico de doença do refluxo gastroesofágico, congestão pulmonar por in- suficiência cardíaca, asma noturna ou síndrome do pânico.

Hábitos e vícios como o uso de álcool e de sedativos, também predispõem as VAS ao colapso no sono porque diminuem o "drive" neuromuscular, enquanto o tabaco o faz, por meio da irritação da mucosa nasal e faringeana reduzindo o calibre da via aérea.

\section{7- IMPACTO DAS APNÉIAS OBSTRUTIVAS DO SONO SOBRE A FUNÇÃO CARDIOR- RESPIRATÓRIA}

O sono interfere drasticamente sobre a função cardíaca e pulmonar de cardiopatas e pneumopatas agravando o desempenho desses órgãos ${ }^{22,23}$. O paciente com doença pulmonar crônica sofre essencialmente os efeitos da hipoventilação induzida pelo sono que agravam sensivelmente as trocas gasosas contribuindo para a hipertensão vascular pulmonar e conseqüente cor pulmonale; por outro lado, soma-se ao aumento do trabalho da caixa torácica e do pulmão hiperinsuflados, um agravamento do desempenho do aparelho respiratório determinado pela posição deitada, gerando mais desconforto respiratório e dispnéia $^{24,25}$. O sono do paciente com DPOC, por exemplo, é fragmentado e superficial. A sobreposição de apnéias do sono ao quadro de DPOC acentua sobremaneira a desvantagem proporcionada pelo sono ${ }^{26} \mathrm{e}$ acelera o processo de hipertensão pulmonar que é o principal fator de redução da sobrevida do pneumopata.

Da mesma forma, aumento de atividade nervosa simpática e parassimpática induzidas pelo sono REM são acompanhadas de importantes flutuações e pausas no ritmo cardíaco que colocam pacientes com doença cardíaca, sob risco aumentado de arritmias, isquemia miocárdica e infarto ${ }^{23}$. Durante o sono NREM, existe em um coração muito comprometido, um potencial para hipotensão que pode piorar o fluxo sanguíneo através das coronárias estenosadas favorecendo isquemia miocárdica e infarto ${ }^{27}$. A coexistência de SAHOS e doença coronariana está associada a risco aumentado de agravamento cardiovascular ${ }^{21}$. A consequiência cardiovascular da SAHOS pode ser aguda ou crônica; o aumento do esforço respiratório durante o colapso faringeano produz uma diminuição substancial na pressão intratorácica a qual contribui para aumento da pré-carga cardíaca e pós-carga ventricular esquerda. Ao término de um episódio de apnéia pode haver aumento do volume sistólico sobre uma vasoconstrição simpática que pode elevar inten- 
samente a pressão arterial durante a noite ${ }^{28}$. Cronicamente a SAHOS pode levar a períodos de hipertensão arterial sistêmica sustentada, cujo mecanismo provavelmente inclui: excitação simpática, reduzida atividade parassimpática e liberação de endotelina ${ }^{29}$. A combinação de efeitos hemodinâmicos agudos e crônicos da SAHOS tem sido associada a risco aumentado de infarto do miocárdio, acidente cérebro vascular e insuficiência cardíaca congestiva.

\section{8- ESCALA DE AVALIAÇÃO DE SONOLÊN- CIA DE EPWORTH}

A avaliação da sonolência deve ser feita pelo clínico antes de decidir pela solicitação de exame polissonográfico, bem como um interrogatório dirigido para outras possíveis causas de sonolência, quando o score da escala somar 10 pontos ou mais.

A escala de sono de Epworth consiste de 8 questões testes que avaliam o grau de sonolência, pontuando cada questão de 0 a 3 de acordo com a menor ou maior probabilidade de ficar sonolento em determinadas situações comuns, podendo totalizar de 0 a 24 pontos, com menor pontuação indicando menos sonolência ${ }^{19}$. É de fácil e rápida aplicação, tem sido validada em diversos estudos clínicos e correlaciona bem com medidas objetivas de sonolência como o teste das múltiplas latências, por exemplo.

\section{Escala de sonolência de Epworth}

Pergunte ao paciente: em face das seguintes situações você tem nenhuma chance de cochilar (0) pequena chance (1), media chance (2) ou grande chance (3)?

\section{Situação}

Sentado lendo

Assistindo TV

Parado num lugar público

Como passageiro de carro por uma hora

Deitado tranqüilo à tarde

Conversando com alguém

Sentado após uma refeição sem álcool

Em um carro parado sob a sinaleira

\section{9- APNÉIAS DO SONO E OBESIDADE}

Há uma relação estreita e recíproca entre os distúrbios respiratórios do sono e obesidade. Cerca de $70 \%$ dos adultos com SAHOS são, pelo menos, sobrepeso. Por causa do depósito de gordura parafaringeana, o obeso pode ter orofaringe estreita e deformada, predisposta ao colapso. Lesão local por vibração das partes moles dessa região durante o sono e ação da pressão negativa inspiratória, anormalmente alta nas vias aéreas estreitas contribuem significativamente para agravamento da oclusão no sono. Cerca de $40 \%$ a $50 \%$ dos obesos mórbidos tem apneias do sono. Em contrapartida a SAHOS tem sido responsabilizada por aumento da resistência a leptina e a insulina ${ }^{30}$ constituindo assim um fator de perpetuação do ciclo de eventos no obeso. O extremo mais grave do espectro dos distúrbios respiratórios do sono é representado pela síndrome de hipoventilação por obesidade ou síndrome de Pickwick, que é associada à morbidade severa e mortalidade muito alta ${ }^{11,31}$.

\section{0- MÉTODO DE DIAGNÓSTICO}

Um exame destinado ao diagnóstico dos distúrbios respiratórios do sono deve permitir a identificação e quantificação (número e duração) dos eventos respiratórios anormais, seus efeitos sobre a saturação da hemoglobina, sua repercussão sobre a freqüência e o ritmo cardíaco e sua relação com os diversos estágios do sono. A polissonografia é considerada o "padrão de ouro" para diagnóstico. Um único exame noturno é satisfatório. Em situações de distúrbio grave é possível firmar o diagnóstico e a gravidade com apenas algumas horas de registro, o que permitiu a proposta do exame em "noite dividida" 32 . É uma maneira econômica, sem perder a eficiência, de fazer o diagnóstico e ajuste da pressão do aparelho de pressão positiva nas vias aéreas, CPAP, em uma única noite (ver Capítulo VI sobre tratamento com CPAP, BiPAP e auto-CPAP).

\section{1- CRITÉRIOS DE DIAGNÓSTICO E DE GRAVIDADE}

As apneias/hipopnéias do sono fazem parte do espectro de distúrbios respiratórios do sono cujos limites entre o normal e o patológico ainda não estão estabelecidos com precisão. A prevalência está aumentando em função do crescente reconhecimento de paci- 
entes com esse problema e pela crescente ubigüi-dade dos fatores de risco principalmente a obesidade e a idade. A importância clínica do problema pode ser julgada pela prevalência e gravidade das consequiências. Em decorrência desses fatores os critérios diagnósticos continuam mudando. Adotamos atualmente no nosso Laboratório os critérios diagnósticos da American Academy of Sleep Medicine ${ }^{12}$, que estabelece o diagnóstico de síndrome das apnéias/hipopnéias obstrutivas do sono em face da presença de 5 ou mais eventos obstrutivos por hora de sono, associada a sintomas relativos a hipersonolência diurna que não podem ser explicados por outros distúrbios. A soma de dois ou mais dos seguintes sintomas também faz parte dos critérios diagnósticos: sensação de sufocação durante o sono, relatos de despertares recorrentes, sensação matinal de noite mal dormida, fadiga diurna e falta de concentração.

O diagnóstico da gravidade da SAHS é estabelecido de acordo com o número de eventos obstrutivos (apnéias, hipopnéias e despertares por esforço respiratório aumentado) por hora da sono. Segundo os critérios o distúrbio é considerado:

$$
\begin{aligned}
& \text { Leve - IAH }(\text { ou IDR }) \geq 5 \text { e } \leq 15 / H ; \\
& \text { Moderado - IAH (ou IDR) }>15 \text { e } \leq 30 / H ; \\
& \text { Grave - IAH }(\text { ou IDR })>30 / H \text {. }
\end{aligned}
$$

\section{2- DIAGNÓSTICO DIFERENCIAL}

Quando falamos de distúrbios respiratórios do sono, outra síndrome se apresenta como de grande importância clínica no diagnóstico diferencial com a SAOS: a síndrome de resistência nas vias aéreas superiores (SRVAS), que é caracterizada por limitação ao fluxo aéreo e aumento da resistência das vias aéreas superiores, levando a despertares repetidos, resultando em fragmentação do sono. Estas alterações ocorrem na ausência de apnéias e dessaturações significativas da hemoglobina. O termo SRVAS foi aplicado inicialmente em pacientes que apresentavam sonolência excessiva diurna sem causa aparente. Estes pacientes foram inicialmente classificados como portadores de hipersonia idiopática, porém quando avaliados com manometria esofágica e pneumotacógrafo, eles apresentavam aumento da resistência das vias aéreas superiores indicada por aumento da pressão negativa intratorácica. A importância de separar a SAHOS da SRVAS é um aspecto controverso. O aspecto importante é estar consciente de que em determinados contextos clínicos, um paciente sintomático que não preenche os critérios diagnósticos para SAHOS talvez esteja acometido por SRVAS, que hoje tem indicações de tratamento semelhantes as dos pacientes com apnéia obstrutiva do sono.

\section{3- TRATAMENTO}

O manejo terapêutico das apnéias obstrutivas do sono inclui medidas gerais como interferência sobre o excesso de peso; evitar bebidas alcoólicas, sedativos e tabaco; adotar no leito posições de decúbito lateral, principalmente quando a polissonografia evidencia relação dos eventos respiratórios anormais com o decúbito dorsal.

O controle da obesidade constitui-se o maior desafio no controle da síndrome, mas precisa ser encarado com todos os recursos clínicos e cirúrgicos, disponíveis (ver Capítulos IX e X sobre tratamento da obesidade).

A avaliação e tratamento de diferentes causas de obstrução nasal é necessária como parte da avaliação geral de pacientes com SAOS e, muitas vezes indispensável à boa adaptação do paciente ao aparelho de pressão positiva nas vias aéreas - CPAP.

As medidas específicas de tratamento são de três modalidades:

- a principal diz respeito ao uso de CPAP (ver Capítulo V);

- outras medidas são cirúrgicas, removendo obstáculos mecânicos das vias aéreas superiores ou remodelando a região faringeana por meio de procedimento sobre a base da língua, o palato mole e as paredes laterais da orofaringe (ver Capítulo VII); outra modalidade cirúrgica diz respeito a correção de defeitos maxilo-mandibulares que corrigem sensivelmente a predisposição ao colapso (ver Capítulo VIII sobre cirurgia de avanço maxilo-mandibular);

- os dispositivos intraorais também têm seu papel na prevenção do colapso da faringe durante o sono, em alguns pacientes selecionados; eles atuam promovendo avanço da mandíbula pelo uso de aparelhos adaptados às arcadas dentárias, aumentando o espaço retrolingual. 
Silva GA, Giacon LAT. Obstructive Sleep Apnea/Hypopnea Syndrome. Medicina (Ribeirão Preto) 2006; 39 (2): 185-194.

ABSTRACT: Respiratory sleep disorders are a relatively new topic in Medicine, its management being multidisciplinary. Its importance is raising since the early $1980^{\text {th }}$ when the diagnostic and therapeutic tools started to become largely available. That made it possible to convincingly show the role of sleep and sleep disorders in raising morbidity and mortality. The prevalence has also raise since the first epidemiologic studies in adults mostly because of risk factors like obesity and age rise. The sleep apnea/hypopnea syndrome is the most important and common respiratory sleep disorder, being about $80 \%$ of those cases that look for care at Clinics of Sleep Study.

Keywords: Apnea. Sleep. Snoring. Disorders of Excessive Somnolonce. Sleep Apnea Syndromes. Sleep Apnea, Obstrutive.

\section{REFERÊNCIAS}

1 - Dement WC. History of sleep physiology and Medicine.In: Krieger MH, Roth T, Dement WC, ed. Principles and practice of sleep medicine. 4th ed. Philadelphia: Elsevier Saunders;2005.p.1066-80

2 - Aserinsky E, Kleitman N. Regularly occurring periods of eye motility, and concomitant phenomena, during sleep. Science 1953;118:273-4.

3 - Dement W, Kleitman N. Cyclic variations in EEG during sleep and their relation to eye movements, body motility, and dreaming. Electroencephalogr Clin Neurophysiol 1957:9:67390.

4 - Gastaut H, Tassinari C, Duron B. Etude polygraphique des syndrome de Pickwick. Rev Neurol 1965;112:568-79.

5 - Jung R, Kuhlo W. Neurophysiological studies of abnormal night sleep and the Pickwickian syndrome. Prog Brain Res 1965;18:140-59.

6 - Lugaresi E, Coccagna G, Mantovani M, Cirignotta F, Ambrosetto G, Baturic P. Hypersomnia with periodic breathing: periodic apneas and alveolar hypoventilation during sleep. Bull Physiopathol Respir 1972;8(5):1103-13.

7 - Guilleminault C, Dement W. 235 cases of excessive daytime sleepness: Diagnosis and tentative of classification. J Neurol Sci $1977 ; 31: 13-27$

8 - Fujita S, Conway W, Zorick F, Roth T. Surgical correction of anatomic abnormalities in obstructive sleep apnea syndrome: Uvulopalatopharyngoplasty. Otolaryngol Head Neck Surg 1981;89:923-34.

9 - Sullivan CE, Issa FG, Berthon-Jones M, Eves L. Reversal of obstructive sleep apnea by continuous positive airway pressure applied through the nares. Lancet 1981;1:862-5.
10 - Bixler EO, Vigontzas AN, Ten Have T, Tyson K, Kales A. Effects of age on sleep apnea in men: I. Prevalence and severity. Am J Respir Crit Care Med 1998;157: 144-8.

11 - Lavie P, Herer P, Peled R, Berger I, Yoffe N, Zomer J, Rubin $\mathrm{AH}$. Mortality in sleep apnea patients: a multivariate analysis of risk factors. Sleep 1995;18:149-57

12 - American Academy of Sleep Medicine Task Force. Sleeprelated breathing disorders in adults: Recommendations for syndrome definition and measurements techniques in clinical research. Sleep 1999;22:667-89.

13 - Shahar E, Whitney CW, Redline S, Lee ET, Newman AB, Javier Nieto F, O'Connor GT, Boland LL, Schwartz JE, Samet JM. Sleep-disordered breathing and cardiovascular disease: Cross-sectional results of Sleep Heart Health Study. Am J Respir Crit Care Med 2001;163:19-25.

14 - Malhotra A, White DP. Obstructive sleep apnoea. Lancet 2002;360:237-45

15 - Fogel RB, Malhotra A, Pillar G, Edwards JK, Beauregard J, Shea SA, White DP. Genioglossal activation in patients with obstructive sleep apnea versus control subjects: mechanisms of muscle control. Am J Respir Crit Care Med 2001;162:2025-30.

16 - Deegan PC, McNicholas WT. Pathophysiology of obstructive sleep apnoea. Eur Respir J 1995;8:1161-78.

17 - Remmers JE, deGroot WJ, Sauerland EK, Anch AM. Pathogenesis of upper airway occlusion during sleep. J Appl Physiol 1978;44:931-8.

18 - Peter JH, Koehler U, Grote L, Podszus T. Manifestations and consequences of obstructive sleep apnoea. Eur Respir J 1995;8:1572-83.

19 - Johns MW. A new method for measuring daytime sleepiness: the Epworth Sleepiness Scale. Sleep 1991;14:540-5. 
20 - Peppard P, Young T, Palta M, Skatrud J. Prospective study of the association between sleep disordered breathing and hypertension. N Eng J Med 2000;342:1378-84.

21 - Hung J, Whitford EG, Parsons RW, Hillman DR. Association of sleep apnoea with myocardial infarction in men. Lancet 1990;336:261-4.

22 - Hypercapnic ventilatory response in sleeping adults. Am Rev Respir Dis 1982;126: 758-62.

23 - Mancia G. Autonomic modulation of the cardiovascular system during sleep. N Engl J Med 1993;328:347-9.

24 - Coccagna G, Lugaresi E. Arterial blood gases and pulmonary and systemic arterial pressure during sleep in chronic obstructive pulmonary disease. Sleep 1978; 1:117-24.

25 - Pierce AK, Jarrett CE, Werkle G Jr, Miller WF. Respiratory function during sleep in patients with chronic obstructive lung disease. J Clin Invest 1966;45:631-6.

26 - Kessler R, Chaouat A, Schinkewitch P, Faller M, Casel S, Krieger J, Weitzenblum E. The obesity-hypoventilation syndrome revisited. Chest 2001:120:369-76.

27 - Andrews TC, Fenton T, Toyosaki N, Glasser SP, Young PM, MacCallum G, Gibson RS, Shook TL, Stone PH. Subsets of ambulatory myocardial ischemia based on heart rate activity. Circadian distribution and response to anti-ischemic medication. The Angina and Silent Ischemia Study Group (ASIS) Circulation 1993;88:92-100.
28 - Weiss JW, Remsburg S, Garpestad E, Ringler J, Sparrow D, Parker JA. Hemodynamic consequences of obstructive sleep apnea. Sleep 1966;19:388-97.

29 - Phillips BG, Narkiewicz K, Pesek CA, Haynes WG, Diken ME, Somers VK. Effects of obstructive sleep apnoea on endothelin-1 and blood pressure. J Hypertens 1999;17:616.

30 - Vgontzas AN, Bixler EO, Chrousos GP. Metabolic disturbances in obesity versus sleep apnoea: the importance of visceral obesity and insulin resistance. J Intern Med 2003;254:32-44.

31) Kress JP, Pohlman AS, Alverdy J, Hall JB. The impact of morbid obesity on oxygen cost of breathing (VO2RESP) at rest. Am J Respir Crit Care Med 1999;160:883-6

31 - , Sanders MH, Costantino JP, , Stiller RA, Atwood CW Jr. Splitnight studies for the diagnosis and treatment of sleep-disordered breathing. Sleep 1996;19(Suppl 10):S255-9. 